\title{
Optimization of Stacking Sequence of Composite Laminates for Optimizing Buckling Load by Neural Network and Genetic Algorithm
}

\author{
M. H. Hajmohammad', M. Salari' ', S. A. Hashemi' and M. Hemmat Esfe ${ }^{2^{*}}$ \\ 'Department of Mechanical Engineering, Imam Hossein University, Tehran, Iran; \\ hadi.hajmohammad@gmail.com,m.salari@gmail.com \\ 2Department of Mechanical Engineering, Najafabad Branch, Islamic Azad University, \\ Isfahan, Iran; m.hemmatesfe@gmail.com
}

\begin{abstract}
Composite beams, plates and shells are widely used in the aerospace industry because of their advantages over the commonly used isotropic structures especially when it comes to weight savings. Buckling analyses of composite structural components must be performed in order to ensure, for instance, that a composite panel designed to be part of a control surface does not buckle thereby compromising its aerodynamic shape. Optimization of composite structures has been performed in this paper using Genetic algorithm. Genetic algorithm (GA) approaches are successfully implemented for the TSP. The buckling load of composite plate, which is obtained by the Artificial Neural Networks, was used as the fitness function in the GA to find its optimized value by arranging the ply stacking sequence.
\end{abstract}

Keywords: Stacking Sequence, Genetic Algorithms, Composite Laminate, Buckling Load, Neural Networks.

\section{Introduction}

Composite laminates have widespread applications in aerospace structures, and optimizations of the corresponding stacking sequences are indispensable. Many of the structures manufactured from these materials such as spars in aircraft wing boxes constitute thin-walled shells and are prone to failure by buckling as such. Therefore, It is essential that the onset of this buckling be predicted, and the effect of buckling on the load carrying capacity of the structure be understood and quantified.

Until now, the problem has been mainly addressed using gradient-based optimization techniques. Recently, it has been studied using evolutionary algorithms, which are optimization tools that do not make use of the gradient of the objective function. Genetic algorithm (GA) is the most common choices studied in literature. GA is an implementation of the rules stated by the Darwin's theory. This simple algorithm deals with discrete optimization problems.

Genetic algorithms (GAs) are adopted for optimization of the stacking sequences [3-9]. Since GAs are one of the stochastic search approaches, they require several parameter tuning processes to avoid any reduction in the computational performance. In the evaluation of chromosomes, these GAs involve high computational costs.

Researchers applied various optimization methods to a number of different design problems involving composite materials using design variables such as fiber direction, ply thickness, or stacking sequence. As one of the methods, enumeration, evaluating all possible designs, has a restricted applicability; for most problems, it is computationally too expensive and impracticable for many problems. Genetic algorithms (GA) are well suited for stacking sequence optimization, and because of their random nature, they can

*Corresponding author:

M. Hemmat Esfe (m.hemmatesfe@gmail.com) 
produce alternative optima in repeated runs. The first application of the algorithm to a structural design problem was presented by Goldberg and Samtani [1], who solved a well-known weight minimization problem of a 10-bar truss structure. The GA algorithm was also applied to optimization of composite laminates. Sivakumar et al. [2] attempted to design a laminated composite plate in the presence of elliptical cutouts for optimum free-vibration response. Park et al. [3] used a GA for the optimal design of composite laminates which comprises four plies subject to various in-plane loading conditions. Fiber orientations of layers were taken as design variables and the objective function was formulated using Tsai-Hill failure criterion. Kristinsdottir et al. [4] presented a formulation to optimize the design of large composite panels when loads vary over the panel. The aim was to minimize the weight of the panel using the thickness and orientations of layers as design variables. They used a global optimization algorithm, improving Hit-and-Run (IHR). Antonio [5] applied the Newton-Raphson iterative procedure and the arc-length method to optimize geometrically nonlinear composite structures based on load-displacement control. Using the fiber orientations as design variables, Savic et al. [6] tried to maximize beam bending and beam axial stiffness using IHR. Duvaut et al. [7] developed a method for determining the optimal direction and volume fraction of fibers at each point of a structure. The fiber orientation and the fiber volume fraction were assumed to be constant within each element of the model, but they varied from element to element.

Spallino and Thierauf [8] investigated thermal buckling optimization of laminated composite plates subject to thermal loading. The optimal design problem was solved using evolution strategies under strain and ply contiguity constraints. de Faria and de Almeida [9] addressed buckling optimization of composite plates subjected to uncertain thermal and non-uniform mechanical loadings. The loading configuration was assumed to be described by piecewise linear functions along the plate edges. An optimal design of antisymmetric laminates under thermal loads was given by Adali and Duffy [10] for the nonhybrid and hybrid cases. The optimum results were given for simply supported laminates with graphite, boron and glass layers under a uniform temperature change.

Todoroki and Ishikawa [11] used response surface method to approximate fitness function of GA. Topal and Uzman [12] presented optimization of thermal buckling load by Modified Feasible Direction method.

\section{Artificial Neural Networks}

Artificial neural networks are model-free intelligent dynamic systems based on experimental data that by processing the data have transmitted hidden laws behind the data to the network structure by processing the data. Artificial neural network based on numerical data or example calculation, learn general rules and try to model the neuro-synaptic structure of human brain.

Artificial neural networks have two basic properties: one, mapping based on experimental data (ability and potency of generalizability) and another one, parallel structurability.

These are suitable and applicable in modeling and simulation of systems, especially in non-linear ones. In other words, artificial neural networks provide appropriate solutions in adaptive systems, particularly when the process to be studied is very complex.

NeUron: the smallest unit of information processing that forms the basis of neural network functions.

Transfer Function: Transfer function $f$ can be linear or non-linear. A transfer function is selected based on solving a specific problem (an issue that is supposed to be solved by neural network).

Network Training: Adjusting the communication weights of neurons per received various examples with the goal of the network output to converge towards the desired output.

\section{Multi-Layer Perceptron (MLP) Neural Networks}

Perceptron neural networks especially multi-layer perceptron, is one of the most practical neural networks. This network is capable of selecting the appropriate number of layers and neurons, which are not often too large, doing the non-linear mapping with arbitrary precision. This is what is proposed as the main solution for data modeling in many engineering issues is proposed as the main solution for data modeling. The neurons in a level, form a layer in a level. Moreover, each layer possesses weight that indicates the effect of two neurons on each other. These networks are fed-forward; it means that each neuron in each layer is connected to all the neurons in preceding layers. These networks are known as interconnected. The mentioned network, actually has been created by joining three single- 
layer perceptrons: one is input layer, middle layer (hidden layer) and the third is output layer. The outputs of first layer, form the input vector of second layer, and so the output vectors of the second layer make the inputs of third layer, and the outputs of the third layer are the desired answer of the network.

Neural networks as learning systems are able to learn from their past, experience and environment and improve their behavior during each learning stage. Improvement in learning during the time should be measured based on the criterion; improvement of criterions models is the target of learning system. Learning law by recursive equations, are generally expressed as differential equations. This recursive equations are called learning laws. Learning law is a process which weights matrix and bias vectors of neural network are set. The aim of learning laws is to train the neural network to perform a specific act, and in other words, artificial neural network during training will be more aware of environment, conditions and aim of its act after any iteration of learning algorithm.

The learning in multi-layer perceptron neural network is done by minimizing mean squares error of output by applying back-propagation learning algorithm and by using numerical iteration methods [13].

\section{Neural Network Properties}

In this study, a three-layers perceptron neural network with 6 neurons ( 5 neurons in 2 hidden layers and one neuron in output layer) was applied for buckling load modeling. General views of this network are shown in Figure 1.

Number of hidden layer neurons was determined by experimental method and with regards to minimum mean squares error (MSE) for prediction of buckling load by neural network as compared with experimental data.

This network is feed-forward and back-propagation technique was used to train the network and weights and biases were determined using Levenberg-Marquardt algorithm. The transfer function employed in the hidden layer is sigmoid (logsig) function while linear function (purelin) was used for outer layer.

Calculations for artificial neural network were implemented in MATLAB (V.2010a 7.10), and were implemented in Sigmaplot (11.0) software for data analyzing data and drawing graph.

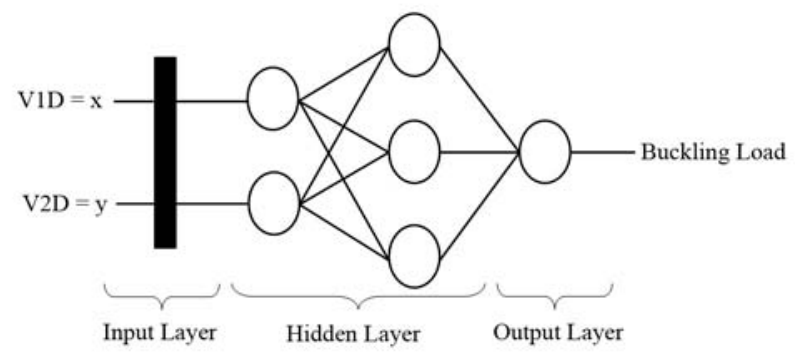

Figure 1. The three-layers perceptron neural network for buckling load modeling.

\section{Approximation of Buckling Function of a Composite Cylinder by MLP Method}

Multi-Layer- Perceptron (MLP) Neural Networks are applied to obtain an approximation to a buckling load of composite cylinder with 10-ply laminates under axial compression loading. The outer radius is $30 \mathrm{~mm}$ and the length of the cylinder is $6 \mathrm{~m}$. Thickness of composite laminate per layer is $0.2 \mathrm{~mm}$.

In this method, determination of the effective variables is important to obtain an approach. So the in-plane and out-of-plane stiffness moduli of symmetric laminates are expressed by means of lamination parameters. A symmetric laminate has four in-plane and four out-of-plane parameters as follows:

$$
\begin{gathered}
A_{i j}=\sum_{k=1}^{N} \overline{Q_{i j}}\left(Z_{k}-Z_{k-1}\right) \\
D_{i j}=\frac{1}{3} \sum_{k=1}^{N} \overline{Q_{i j}}\left(Z_{k}^{3}-Z_{k-1}^{3}\right)
\end{gathered}
$$

$$
V=\frac{1}{t N} \sum_{k=1}^{N} \int_{(N-k) t}^{(N-k+1) t}\left[\begin{array}{c}
\cos 2 \theta \\
\cos 4 \theta \\
\sin 2 \theta \\
\sin 4 \theta
\end{array}\right] d z=\frac{1}{t N} \sum_{k=1}^{N}\{(N-k+1)-(N-k)\}\left[\begin{array}{c}
\cos 2 \theta \\
\cos 4 \theta \\
\sin 2 \theta \\
\sin 4 \theta
\end{array}\right]
$$

$$
W=\frac{3}{t^{3} N^{3}} \sum_{k=1}^{N} \sum_{(N-k) t)}^{(N-k+1)}\left[\begin{array}{c}
\cos 2 \theta \\
\cos 4 \theta \\
\sin 2 \theta \\
\sin 4 \theta
\end{array}\right] z^{2} d z=\frac{3}{t^{3} N^{3}} \sum_{k=1}^{N}\left\{(N-k+1)^{3}-(N-k)^{3}\right\}\left[\begin{array}{c}
\cos 2 \theta \\
\cos 4 \theta \\
\sin 2 \theta \\
\sin 4 \theta
\end{array}\right]
$$


Table 1. Buckling load of composite laminate

\begin{tabular}{|c|c|c|c|c|}
\hline Row & Lay-up & w1 & w2 & Buckling load (N) \\
\hline 1 & {$\left[\begin{array}{l}0_{2} \\
\overline{60} \\
\overline{90} \\
\overline{30}\end{array}\right]_{s y m}$} & $4.37 \mathrm{E}-10$ & $5.07 \mathrm{E}-10$ & 224,350 \\
\hline 2 & {$\left[\begin{array}{c}\frac{0}{60} \\
\frac{90}{30} \\
\frac{30}{0}\end{array}\right]_{s y m}$} & $1.49 \mathrm{E}-10$ & $3.15 \mathrm{E}-10$ & 156,750 \\
\hline 3 & {$\left[\frac{\frac{0}{60}}{\frac{30}{90}}\right.$} & $2.45 \mathrm{E}-10$ & $2.19 \mathrm{E}-10$ & 167,620 \\
\hline 4 & {$\left[\frac{\frac{0}{30}}{\frac{90}{60}}\right.$} & $3.09 \mathrm{E}-10$ & $3.15 \mathrm{E}-10$ & 213,480 \\
\hline 5 & {$\left[\frac{\frac{0}{90}}{\frac{60}{30}}\right.$} & $1.01 \mathrm{E}-10$ & $4.59 \mathrm{E}-10$ & 152,880 \\
\hline 6 & {$\left[\begin{array}{c}\frac{30}{0} \\
\frac{60}{0} \\
\frac{0}{90}\end{array}\right]_{s y m}$} & $3.41 \mathrm{E}-10$ & $2.67 \mathrm{E}-11$ & 182,660 \\
\hline 7 & {$\left[\begin{array}{c}\frac{30}{90} \\
\frac{0}{60} \\
\frac{0}{0}\end{array}\right]_{s y}$} & $5.33 \mathrm{E}-11$ & $1.23 \mathrm{E}-10$ & 137,790 \\
\hline
\end{tabular}


Optimization of Stacking Sequence of Composite Laminates for Optimizing Buckling Load by Neural Network and Genetic Algorithm

8

$\left[\frac{\frac{60}{0}}{\frac{30}{0}}\right]_{s y m}$

9

$\left[\frac{\frac{60}{90}}{\frac{0}{30}} \frac{}{0}\right]_{s y m}$

10

$\left[\frac{\frac{90}{0}}{\frac{30}{0}}\right]_{\text {sym }}$

$\left[\begin{array}{l}\frac{90}{60} \\ \frac{0}{30} \\ 0\end{array}\right]_{s y m}$

$\left[\frac{\frac{30}{30}}{\frac{90}{60}} \frac{}{0}\right]_{s y m}$

$\left[\frac{\frac{30}{60}}{\frac{90}{30}}\right]_{s y m}$

$\left[\frac{\frac{30}{0}}{\frac{90}{30}} \frac{}{60}\right]_{s y m}$

$\left.\frac{\frac{30}{90}}{\frac{60}{30}}\right]_{s y m}$

14

$15 \quad\left[\begin{array}{c}\frac{30}{90} \\ \frac{60}{0} \\ \frac{30}{s y m}\end{array}\right.$
$1.17 \mathrm{E}-10$

2.67E-11

138,160

$-2.35 \mathrm{E}-10$

$1.23 \mathrm{E}-10$

101,700

$-4.27 \mathrm{E}-11$

$5.07 \mathrm{E}-10$

155,130

$-2.99 \mathrm{E}-10$

3.15E-10

97,955

1.47E-10

$-1.73 \mathrm{E}-10$

174,030

2.67E-12

$-1.25 \mathrm{E}-10$ $\begin{array}{lll}2.75 \mathrm{E}-10 & 1.15 \mathrm{E}-10 & 244,200\end{array}$

$\begin{array}{lll}-4.53 \mathrm{E}-11 & 1.87 \mathrm{E}-11 & 131,040\end{array}$ 


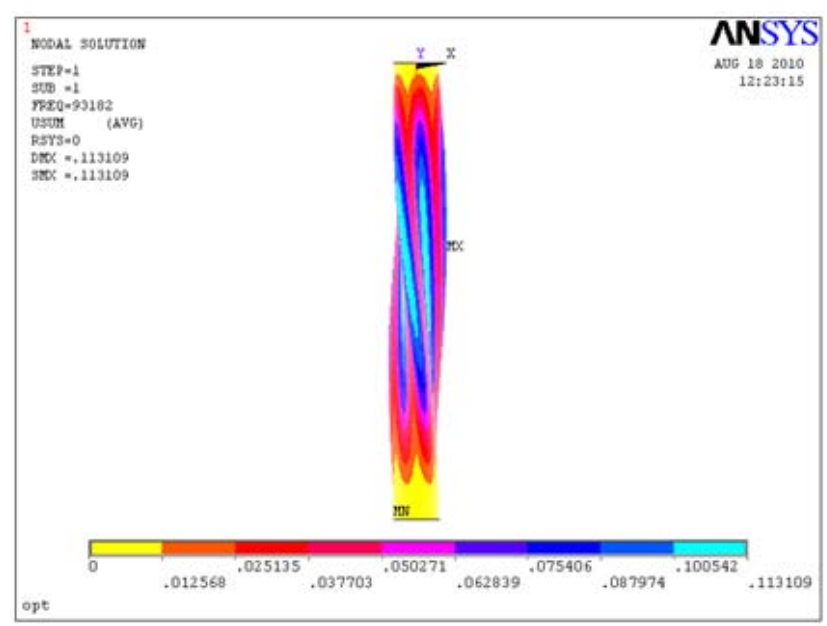

Figure 2. Buckling of composite laminate by 10-ply laminates $[90 / 60 / 30 / 0(2)]$ sym.

The effective variables for the composite cylinder with constant thickness are defined as follows:

$$
W=\frac{3}{t^{3} N^{3}} \sum_{k=1}^{N}\left\{(N-k+1)^{3}-(N-k)^{3}\right\}\left[\begin{array}{l}
\cos 2 \theta \\
\cos 4 \theta
\end{array}\right]
$$

The design of experiments requires 15 FEM analyses to be performed. The selected laminates and buckling load that should be obtained from Eq.(5) in term of two variables $\mathrm{w}_{1}$ and $\mathrm{w}_{2}$ are shown in Table 1.

Critical buckling load is shown in Figure 2 and is equal to $93182 \mathrm{~N}$.

\section{Modeling and Discussion}

In the present study, for buckling load modeling was used an experimental data set including W1, W2 and buckling load experimental values were used for buckling load modeling. This data set as training data to a three- layer perceptron neural network with properties as expressed in the previous section. At the end of training stage, the neural network with mapping based on experimental data has transmitted hidden laws behind the data to the network structure and fitted a model between the input and output data. The resulting model, fitted the experimental data with high accuracy and the predicted values for buckling load possesses regression $\mathrm{R}=0.99509$ in comparison with experimental values. Figure 3 shows the regression graph

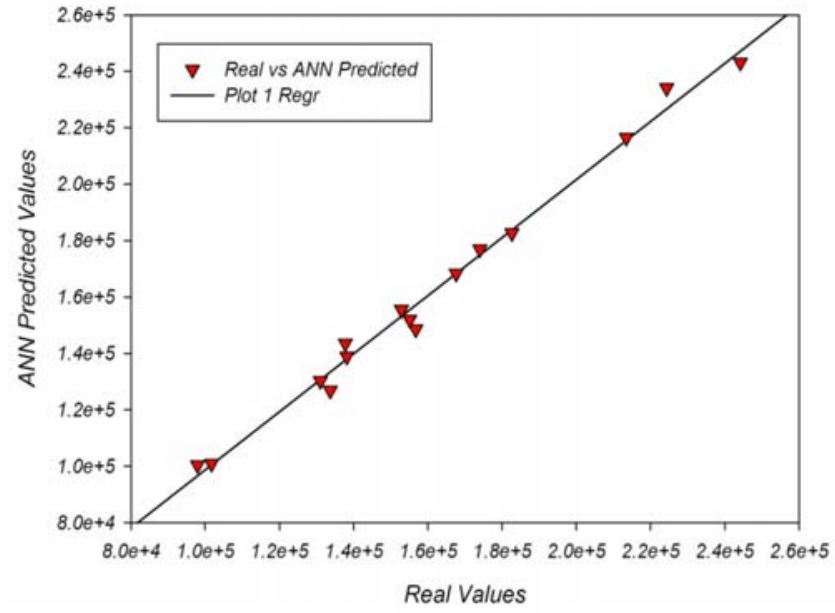

Figure 3. The comparison of neural network predicted values and experimental values.

of predicted values for buckling load in comparison with experimental values.

\section{The Genetic Algorithms}

\subsection{Generalities}

The Genetic Algorithm (GA) is a stochastic global search method that mimics the metaphor of natural biological evolution. GA operates on a population of potential solutions applying the principle of survival of the fittest to produce (hopefully) better and better approximations to a solution. At each generation, a new set of approximations is created by the process of selecting individuals according to their level of fitness in the problem domain and breeding them together using operators borrowed from natural genetics. This process leads to the evolution of populations of individuals that are better suited to their environment than the individuals that were created from, just as in natural adaptation. The fitness function establishes the basis for selection of pairs of individuals that will be mated together during reproduction.

During the reproduction phase, each individual is assigned a fitness value derived from its raw performance measure given by the objective function. This value is used in the selection to bias towards more fitted individuals. Highly fitted individuals, relative to the whole population, have a high probability of being selected for mating whereas less fitted individuals have a correspondingly low probability of being selected. 
Once the individuals have been assigned a fitness value, they can be chosen from the population, with a probability according to their relative fitness, and recombined to produce the next generation. Genetic operators manipulate the characters (genes) of the chromosomes directly, using the assumption that certain individual's gene codes, on average, produce more fitted individuals. The recombination operator is used to exchange genetic information between pairs or larger groups of individuals [14].

\subsection{Stacking Sequence of Composite}

\section{Laminates}

In this problem, a desired sequence has maximum critical buckling load. In this paper, we consider this problem as Traveling Salesman Problem (TSP). TSP is a typical example of a very hard combinatorial optimization problem. Consider a graph which shows some cities and rods among them. In this graph, $G=(V, E), V$ is set of vertices each being a city. The edge set $\mathrm{E}$ of graph is relation among cities. The problem is to find the shortest tour that passes through each vertex exactly once in a given graph. Tour is a complete permutation of vertices such that two successive city in this permutation can be connected by an edge. If you consider a complete graph, then all permutation of nodes are possible tours.

In our problem, there is a sequence or permutation of Composite laminates with maximum critical buckling load. In both problems, search algorithm has to find a permutation that has optimum value of a fitness function. Consider a permutation $\mathrm{P}$ that shows the sequence of laminate. Fitness Function in GA has to calculate the critical buckling load according to $\mathrm{P}$ and yield a single value.

\subsection{Application of GA for Maximization of Critical Buckling Load}

We have to identify some aspects of GA for applying them on our problem: chromosome representation, crossover operator, mutation operator and selection method. Before implementation, some parameters must be configured: population size, crossover rate, mutation probability, etc. The integer array is used to encode a chromosome in GA. This array has to be a permutation of 1 to $n$. $n$ is the number of laminate. For making cross between two chromosomes, cyclic crossover is used. In this crossover, inherent data of a child on a cycle start with the first parent and completed by the second parent. Swap mutation is used as mutation operator. This operator swaps two elements of a chromosome and produces mutated chromosome [15].

\section{Optimization of Composite Laminate Shell with 10-ply Laminates to Maximize Critical Buckling Load by Genetic Algorithm and Multi-Layers Perceptron (MLP) Neural Networks}

Optimization with genetic algorithm needs a fitness function. This function for a composite shell is represented by Multi-Layers Perceptron (MLP) Neural Networks.

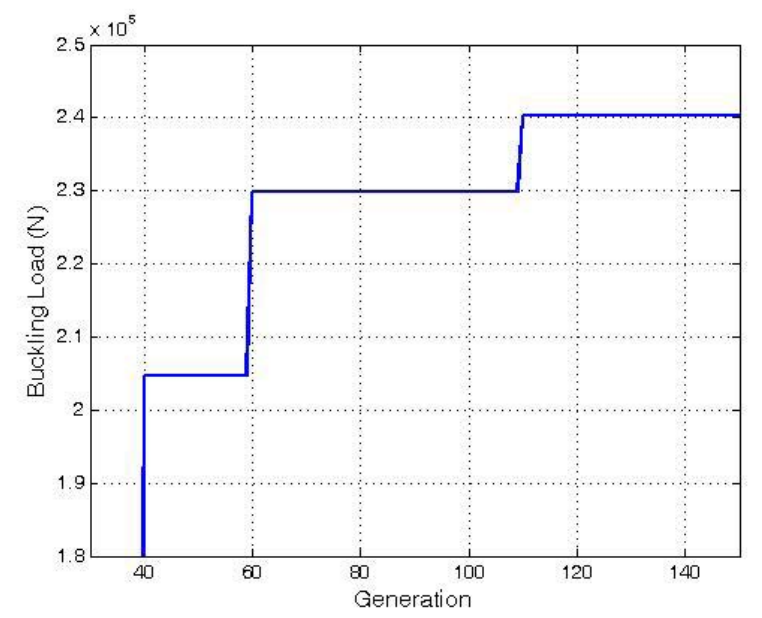

Figure 4. Buckling load in optimization process.

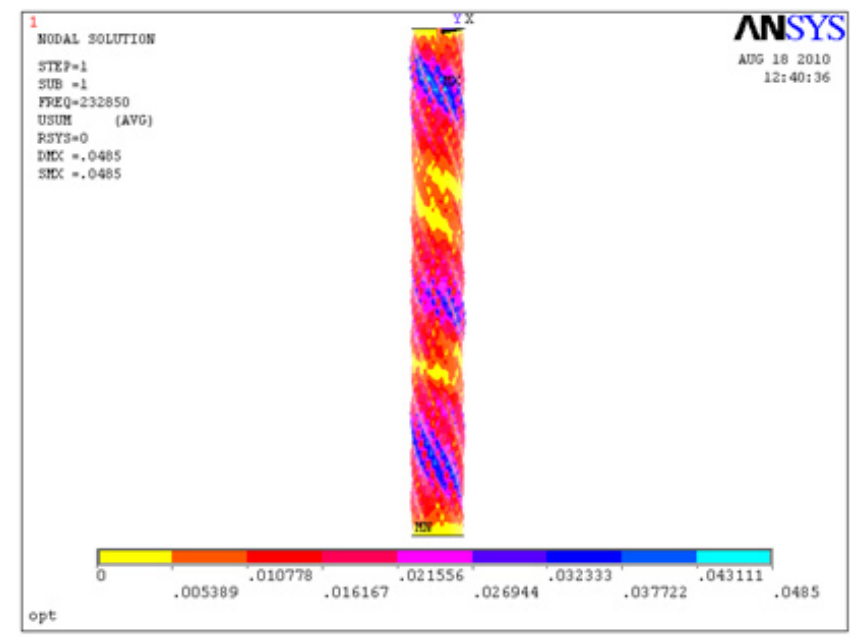

Figure 5. Analysis of optimized buckling load. 
Properties of an orthotropic graphite-epoxy are considered. The composite shell has 10-ply graphite -epoxy laminates and variables of fitness function are based on Eq.5.

Optimization process as shown in Figure 4 starts by selecting angles as [90/60/30/0(2)]s. Critical buckling load for this angles is $93182 \mathrm{~N}$ as shown in Figure 1. Optimized data has been obtained in genetic algorithm process and optimum answer is stacking sequence of [0(2)/30/60/90] s. This optimization is performed for 150 generations and optimized buckling load has been obtained as $232850 \mathrm{~N}$ as shown in Figure 5.

\section{Conclusion}

This paper presents a new approach to optimal design of the laminated shells subjected to the axial compressive loading. Optimized buckling load that is calculated by Multi-Layers Perceptron (MLP) and Neural Networks function is $240300 \mathrm{~N}$ and has admissible value than experimental value.

After determining an objective function, genetic algorithm was employed to obtain an optimal design. Optimization results showed that diversity of stacking sequence could increase buckling load from $93182 \mathrm{~N}$ to $232850 \mathrm{~N}$. Results showed that combining the Neural Networks and genetic algorithm provided an effective tool to optimize the stacking sequence of composite laminates.

\section{References}

1. Goldberg D E, Samtani M P (1986). Engineering optimization via genetic algorithm, Proceedings of the Ninth Conference on Electronic Computation, ASCE, 471-482.

2. Sivakumar K, Iyengar N G R et al. (1998). Optimum design of laminated composite plates with cutouts using a genetic algorithm. Composite Structures, vol 42(3), 265-279.
3. Park J H, Hwang J H et al. (2001). Stacking sequence design of composite laminates for maximum strength using genetic algorithms, Composite Structures, vol 52(2001), 217-231.

4. Kristinsdottir B P, Zabinsky Z B et al. (2001). Optimal design of large composite panels with varying loads, Composite Structures, vol 51(1), 93-102.

5. Antonio C A (1999). Optimizations of geometrically nonlinear composite structures based on load-displacement control, Composite Structure, vol 46(4), 345-356.

6. Savic V, Tuttle M E et al. (2001). Optimization of composite I-sections using fiber angles as design variables, Composite Structure, vol 53(3), 265-277.

7. Duvaut G, Terrel G et al. (2000). Optimization of fiber reinforced composites, Composite Structure, vol 48(1-3), 83-89.

8. Spallino R, and Thierauf G (2000). Thermal buckling optimization of composite laminates by evolution strategies. Computers \& Structures, vol 78(5), 691-697.

9. de Faria A R, and de Almeida S F M (2004). Buckling optimization of composite plates subjected to uncertain thermal and nonuniform mechanical loadings, Inverse Problems, Design and Optimization Symposium Rio de Janeiro, Brazil.

10. Adali S, and Duffy K J (1990). Optimal design of antisymmetric hybrid laminates against thermal buckling, Journal of Thermal Stresses, vol 13(1), 57-71.

11. Todoroki A, and Sekishiro M (2007). New iteration fractal branch and bound method for stacking sequence optimizations of multiple laminates, Composite Structures, vol 81(3), 419-426.

12. Topal U, and Uzman U (2008). Thermal buckling load optimization of laminated composite plates, Thin-Walled Structures, vol 46(6), 667-675

13. Graupe D (2007). Principles of artificial neural networks, vol 6, $2^{\text {nd }}$ Edition, World Scientific Publishing Co. Pte. Ltd.

14. Falkenauer E (1997). Genetic Algorithms and Grouping Problems, Chichester, England: John Wiley \& Sons Ltd, ISBN 978-0-471-97150-4.

15. Syswerda G (1989). Uniform crossover in genetic algorithms, J. D. Schaffer. Proceedings of the Third International Conference on Genetic Algorithms, Morgan Kaufmann. 